\title{
Induced Chern-Simons-like action in Lorentz-violating massless QED
}

\author{
${ }^{a}$ F.A. Brito, ${ }^{b}$ L.S. Grigorio, ${ }^{b}$ M.S. Guimaraes, ${ }^{a, c}$ E. Passos, ${ }^{b}$ C. Wotzasek \\ ${ }^{a}$ Departamento de Física, \\ Universidade Federal de Campina Grande, Caixa Postal 10071, \\ 58109-970 Campina Grande, Paraíba, Brazil \\ ${ }^{b}$ Instituto de Física, Universidade Federal do Rio de Janeiro, \\ Caixa Postal 21945, Rio de Janeiro, Brazil \\ ${ }^{c}$ Departamento de Física, \\ Universidade Federal da Paraíba, Caixa Postal 5008, \\ 58051-970 João Pessoa, Paraíba, Brazil
}

\begin{abstract}
In the present work, we study different aspects of Lorentz and CPT symmetry violation in extended massless QED. By following the observation that the 2+1 dimensional Maxwell-ChernSimons theory can be originated from the $3+1$ dimensional Chern-Simons-like action, we also focus on the fermion sector to relate the $3+1$ dimensional extended massless QED to $2+1$ dimensional massive QED. We take advantage of this to state that the Chern-Simons-like action in extended massless QED can be induced with its coefficient being well defined and finite just as its $2+1$ counterpart. We make use of three different regularization schemes by inducting the Chern-Simons-like in $3+1$ dimensions to support the conjecture.
\end{abstract}

PACS numbers: 11.30.Er, 11.10.Wx, 12.20.Ds

\section{INTRODUCTION}

The main goal of this investigation is to revisit the question posed by the ambiguity problem of the ChernSimons-like action induced by radiative corrections in the photon sector of the Lorentz-violating extended Standard Model [1]. Several theoretical investigations have pointed out the possibility of the Lorentz and CPT symmetries being violated in nature 1, 2, 3, 4, 5, 6, 7, 8, 9, 10, 11, 12, 13, 14, 15, 16, 17]. These symmetries would then be approximately realized. A great body of phenomenological results was obtained by realizing that this violation can indeed be generated in the photon sector of QED by adding the Chern-Simons-like action $\mathcal{L}_{C S}^{3+1}=\frac{1}{2} k_{\mu} \epsilon^{\mu \alpha \beta \gamma} F_{\alpha \beta} A_{\gamma}$, with $k_{\mu}$ being a constant quadrivector characterizing a preferred direction of the space-time. As one knows, this extended electromagnetism does not break the gauge symmetry of the action and equations of motion but it does modify the dispersion relations for different polarization of photons. The Chern-Simons-like action is known to have some important implications, such as birefringence of light in the vacuum [9]. Many interesting investigations in the context of Lorentz-CPT violation have appeared recently in the literature. For instance, several issues were addressed, such as Čerenkov-type mechanism called "vacuum Cerenkov radiation" to test the Lorentz symmetry [18], changing of gravitational redshifts for differently polarized Maxwell-Chern-Simons photons [19], evidence for the Lorentz-CPT violation from the measurement of CMB polarization [20], supersymmetric extensions [21], breaking of the Lorentz group down to the little group associated with $k_{u}[22]$ and magnetic monopoles inducing electric current 23].

A possible modification in the fermionic sector is to consider the CPT-odd term $\bar{\psi} \not \gamma_{5} \psi$ with a constant quadrivector $b_{\mu}$ introducing CPT symmetry breaking. This modification brings one of the most interesting and controversial results regarding the dynamical origin of the parameter $k_{\mu}$ present in the Lorentz and CPT symmetry breaking obtained when we integrate over the fermion fields in the modified Dirac action. The result is the induction of the Chern-Simons-like action via radiative corrections which may lead to a relation between $k_{\mu}$ and $b_{\mu}$, such that the last is given as an input in the theory.

The induction of the Chern-Simons-like action, $\mathcal{L}_{C S}$, is an important result in the study of the Lorentz symmetry violation [1, 4, 8]. This term, naturally emerges as a perturbative correction in the theory suggested in [1] as a possible extension of QED by an axial-vector term

$$
\mathcal{L}=\bar{\psi}(i \not \partial-M) \psi-\bar{\psi} \not \gamma_{5} \psi-e \bar{\psi} \not A \psi
$$

By carrying out the integration over fermions it is possible to obtain a relation between the coefficients $k_{\mu}$ and $b_{\mu}$ in terms of loop integrals as $k_{\mu}=C b_{\mu}$ with some of them being possibly divergent. Therefore since one has to implement a proper regularization to calculate these integrals, the constant $C$ relating the coefficients turns out to be dependent on the regularization scheme used [24]. The ambiguity of the results, manifested in the dependence on the regularization scheme, has been intensively discussed in the literature. Several studies have shown that $C$ can be found to be finite but undetermined [25, 26, 27, 28, 29]. Astrophysical observations impose very stringent experimental bounds on $k_{\mu}$ suggesting that it should vanish. Since the coefficient $k_{\mu}$ of the radiatively induced Chern-Simons-like action depends on $b_{\mu}$ it is natural to expect that the constant $b_{\mu}$ can also suffer an experimental bound in this framework. However, if ambiguities are present there is no way to know for sure to what extent the experimental bounds would constrain the constant $b_{\mu}$, simply because $C$ is un- 
determined. In other words, we cannot define the fate of the constant that is responsible for the Lorentz and CPT violation in the fermion sector by simply measuring $k_{\mu}$. In the following we are going to reduce the well studied Lagrangian (1) to the massless case in attempting to shed some light on the issue of inducing the Chern-Simons-like action with no ambiguities.

The paper is organized as follows. In Sec. III, we show how to relate the $3+1$ dimensional extended massless QED to $2+1$ dimensional massive QED. We also comment on the relation between the induced $3+1$ dimensional Chern-Simons-like term and its counterpart in $2+1$ dimensions. In Sec. III we show how to induce the Chern-Simons-like term in an extended massless QED. In Sec.IV] we make use of the dimensional regularization and the momentum cut-off regularization. In Sec. V , we consider the induction of the Chern-Simons-like term at finite temperature. In this section, we use the limit of zero temperature as our third regularization scheme. Finally, in Sec. VI we present our ending comments.

\section{II. $3+1$ LORENTZ VIOLATION AS THE ORIGIN OF $2+1$ FERMIONIC MASS}

In this section we shall point an interesting motivation to study the $3+1$ dimensional massless QED. The approach we intend to take in the following is based on the well known fact that in $2+1$ QED the massive fermionic degrees of freedom gives rise to the CS term without any ambiguity in its coefficient. It is useful to review this argument here. The physical origin of this phenomenon is the fact that the fermionic mass in $2+1$ dimensions is just a spin density, that is, massive fermions have definite spin in this dimensionality with a fixed orientation given by the sign of the mass term. As result $P$ and $T$ symmetries are broken. So, for instance, if we are interested in a scale well bellow that defined by the fermionic mass, fermions will only contribute through their quantum fluctuations disturbing the electromagnetic propagation. The resulting effective theory must convey the information of the $P$ and $T$ discrete symmetry breaking and hence the induction of the CS term. This term does not depend on the absolute value of the fermionic mass, only on its sign, while all the other corrections are attenuated by the mass which makes the resulting MaxwellChern-Simons (MCS) a suitable effective field theory for QED with very massive fermions.

What is important for the present work is to observe that the MCS theory can be viewed as the dimensionally projected theory [30] of the CPT-odd Carroll-FieldJackiw model, which is just the Maxwell theory augmented by the CPT-odd term discussed previously. This simple observation prompt us to search for a system in $3+1$ dimensions having $2+1$ dimensional QED as its dimensional reduction. An immediate point to call up to attention is that the $P$ and $T$ symmetry breaking mass in $2+1$ dimensions bears no relation to the $3+1$ dimen- sional fermionic mass. In fact it is easily seen that the $2+1$ dimensional fermionic mass term is related to the axial-vector term $\bar{\psi} b \gamma_{5} \psi$ in $3+1$ dimensions. Choosing the $b_{\mu}$ as a space-like vector and in the 3 -direction with $b_{3}=m$ and working in the chiral representation for the gamma matrices

$$
\begin{aligned}
& \gamma^{5}=\left(\begin{array}{cc}
-1 & 0 \\
0 & 1
\end{array}\right) ; \quad \gamma^{\mu}=\left(\begin{array}{cc}
0 & \sigma^{\mu} \\
\bar{\sigma}^{\mu} & 0
\end{array}\right) ; \\
& \sigma^{\mu}=(1, \vec{\sigma}) ; \quad \bar{\sigma}^{\mu}=(1,-\vec{\sigma})
\end{aligned}
$$

we obtain

$$
b_{\mu} \bar{\psi} \gamma^{\mu} \gamma_{5} \psi \rightarrow m\left(\bar{\psi}_{L} \psi_{L}+\bar{\psi}_{R} \psi_{R}\right)
$$

were $\psi_{L}$ and $\psi_{R}$ are the left and right two-component spinor fields, respectively. This result is intuitively clear if we realize that the $P$ and $T$ symmetry breaking generated by the fermionic mass term in $2+1$ is just the dimensional reduction of the Lorentz symmetry breaking in $3+1$ dimensions. The Lorentz violating vector breaks the Lorentz symmetry in $3+1$ dimensions because it defines a preferred direction and a orientation. Upon fixing its direction and projecting the theory on the corresponding orthogonal plane there remains the preferred orientation encapsulated in the sign of $m$ in (3) which is the source of the $P$ and $T$ symmetry breaking in $2+1$ dimensions.

The Dirac equation that follows from (1),

$$
\left(i \not \partial-M-\not b \gamma_{5}-e \not A\right) \psi=0,
$$

can be decomposed in its chiral components as

$$
\begin{gathered}
\left(i \sigma^{\mu} \partial_{\mu}-e \sigma^{\mu} A_{\mu}-\sigma^{\mu} b_{\mu}\right) \psi_{R}-M \psi_{L}=0 \\
\left(i \bar{\sigma}^{\mu} \partial_{\mu}-e \bar{\sigma}^{\mu} A_{\mu}+\bar{\sigma}^{\mu} b_{\mu}\right) \psi_{L}-M \psi_{R}=0 .
\end{gathered}
$$

For $M \rightarrow 0$ these equations decouple as is well known since chiral symmetry is restored. A 'natural' setup considers a dynamical dimensional reduction mechanism via domain wall background. In this point of view $M \equiv m \mp m \tanh \left(\frac{x^{3}}{\Delta}\right) \rightarrow 0$ outside $\left(x^{3} \rightarrow \pm \infty\right)$ the two-dimensional domain wall with thickness $\Delta$, whereas $M \equiv m$ inside the domain wall at $x^{3} \simeq 0$. Thus, the $3+1$ theory outside the domain wall has chiral symmetry but breaks Lorentz and CPT symmetry, while in the $2+1$ theory inside the domain wall the Lorentz and CPT symmetry is restored but the chiral symmetry is broken. Similarly, in this mechanism the induced 3+1 ChernSimons-like term outside the domain wall is expected to match the induced and determined Cherns-Simons term in $2+1$ dimensions inside the domain wall, since the latter can be brought from the former by just decomposing the $3+1$ dimensional fields into $2+1$ dimensional fields, i.e., $A^{\mu} \rightarrow\left(A^{a}, \varphi\right), k^{\mu} \rightarrow\left(k^{a},-k_{3}\right), a=0,1,2$, such as $\mathcal{L}_{C S}^{2+1}=\frac{1}{2} k_{3} \epsilon^{a b c} F_{a b} A_{c}$, up to scalar-vector field coupling. Thus, according to this match, one expects that at least one component of the Chern-Simons-like term in $3+1$ dimensions should be finite and determined. What is interesting though is that by further projecting into 
the plane $x^{3}=0$ and again working with a space like $b_{\mu}$-vector in the 3 -direction with $b_{3}=m-$ see Fig. 1 1 the first equation in (5), say, will read

$$
\left(i \sigma^{a} \partial_{a}-e \sigma^{a} A_{a}-e \sigma^{3} \phi-\sigma^{3} m\right) \psi_{R}=0 .
$$

where $\phi \equiv A_{3}, a=0,1,2$ and $\partial_{3} \psi=0$ (there is no transversal momentum $p_{3}$ ). By choosing an appropriate representation for the Dirac matrices in $2+1$ this equation may be shown to follow from the $2+1$ action

$$
S=\int d^{3} x \bar{\psi}_{R}(\not \not \partial-e \not A-e \phi-m) \psi_{R} .
$$

As already discussed, the action (8) is known to induce a finite and well determined Chern-Simons action in $2+1$. Then, as we anticipate by using a domain wall background point of view, it is reasonable to expect that the proper fermionic action in $3+1$ that will induce the CPTodd term without ambiguity is given by (1) with $M=0$, i.e., an extended massless QED. This is precisely what happens, at least for the three different regularization schemes used, as we shall show in the following sections.

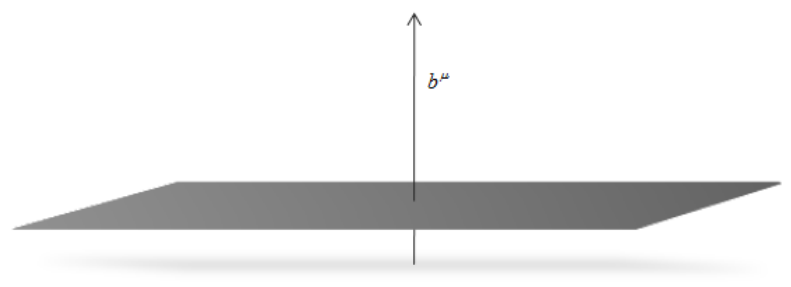

FIG. 1: The space like $b_{\mu}$-vector in the third direction. The $2+1$ dimensional action for the massive QED and ChernSimons term is on the plane.

\section{THE INDUCED CHERN-SIMONS ACTION IN AN EXTENDED MASSLESS QED}

Let us introduce an extension of the usual Lagrangian density in the absence of mass term [10, 15, 31] as follows

$$
\mathcal{L}=\bar{\psi}\left(i \not \partial-\not b \gamma_{5}-e \not A\right) \psi .
$$

The one loop effective action $S_{\text {eff }}[b, A]$ of the gauge field $A_{\mu}$ in this theory can be expressed in the form of the following functional trace

$$
S_{e f f}[b, A]=-i \operatorname{Tr} \ln \left[\not p-\not b \gamma_{5}-e \not A\right] .
$$

Notice that this point is delicate by the fact that the trace symbol-Tr stands for the trace over Dirac matrices, trace over internal space, as well as for the integration in momentum and coordinate spaces. Hence, in this present case the Eq.(10) cannot be directly calculated and written as $S_{\text {eff }}=\int d^{4} x \mathcal{L}$ since the electromagnetic field- $A_{\mu}$ is coordinate dependent and, therefore, does not commute with functions of momentum. In fact, it is not an easy task to separate out the momentum and space dependent quantities, and carry out the integrations in respective spaces. To solve this problem, we will use the method of derivative expansion 32] (see also [24]), and proceed as in the following. This functional trace can be represented as

$$
S_{e f f}[b, A]=S_{e f f}[b]+S_{e f f}^{\prime}[b, A],
$$

where the first term is $S_{e f f}[b]=-i \operatorname{Tr} \ln \left[\not p-\not b \gamma_{5}\right]$, which does not depend on the gauge field, and the only nontrivial dynamics is governed by the second term $S_{\text {eff }}^{\prime}[b, A]$, which is given by the following power series

$$
S_{e f f}^{\prime}[b, A]=i \operatorname{Tr} \sum_{n=1}^{\infty} \frac{1}{n}\left[\frac{1}{\not b-\not b \gamma_{5}} e A\right]^{n} .
$$

To obtain the Chern-Simons-like action we should expand this expression up to the second order in the gauge field

$$
S_{\text {eff }}^{\prime}[b, A]=S_{\text {eff }}^{(2)}[b, A]+\ldots .
$$

The ellipsis in (13) stands for the terms of higher orders in the gauge field. Here

$$
S_{\text {eff }}^{(2)}[b, A]=-\frac{i e^{2}}{2} \operatorname{Tr}\left[S_{b}(p) A S_{b}(p) A\right],
$$

where $S_{b}(p)$ is the $b^{\mu}$-dependent propagator of the theory defined as

$$
S_{b}(p)=\frac{i}{\not p-\not b \gamma_{5}} .
$$

Now, we can use the identity

$$
A_{\mu}(x) S_{b}(p)=S_{b}(p-i \partial) A_{\mu}(x),
$$

with its respective expansion of operators given in the form

$$
S_{b}(p-i \partial)=S_{b}(p)+S_{b}(p) \not \partial S_{b}(p)+\cdots
$$

Substituting this back into the expression (14), we obtain

$$
S_{\text {eff }}^{(2)}[b, A]=\int d^{4} x \Pi^{\mu \lambda \nu} \partial_{\lambda} A_{\mu} A_{\nu},
$$

with the one loop self-energy tensor being given by

$$
\Pi^{\mu \lambda \nu}=-\frac{i e^{2}}{2} \int \frac{d^{4} p}{(2 \pi)^{4}} \operatorname{tr}\left[S_{b}(p) \gamma^{\mu} S_{b}(p) \gamma^{\lambda} S_{b}(p) \gamma^{\nu}\right],
$$

where tr, means that the trace just acts over the gamma matrices. After some algebraic manipulations, we can rewrite the above propagator as

$$
S_{b}(p)=W_{1} P_{L}+W_{2} P_{R},
$$

where

$$
P_{R, L}=\frac{1 \pm \gamma_{5}}{2}
$$


and

$$
W_{1}=\frac{i(p+b)^{2}(\not p-\not b)}{\left(p^{2}-b^{2}\right)^{2}}, W_{2}=\frac{i(p-b)^{2}(\not p+\not b)}{\left(p^{2}-b^{2}\right)^{2}} .
$$

Therefore, the expression to the self-energy tensor in (19) can be also written in the form

$$
\Pi^{\lambda \mu \nu}=-\frac{i e^{2}}{2} \int \frac{d^{4} p}{(2 \pi)^{4}} T^{\mu \lambda \nu}(p),
$$

with

$$
\begin{aligned}
& T^{\mu \lambda \nu}(p)=\operatorname{tr}\left[W_{1} P_{R} \gamma^{\mu} W_{1} P_{R} \gamma^{\lambda} W_{1} P_{R} \gamma^{\nu}+W_{1} P_{R} \times\right. \\
& \gamma^{\mu} W_{1} P_{R} \gamma^{\lambda} W_{2} P_{L} \gamma^{\nu}+W_{1} P_{R} \gamma^{\mu} W_{2} P_{L} \gamma^{\lambda} W_{1} P_{R} \gamma^{\nu}+ \\
& W_{1} P_{R} \gamma^{\mu} W_{2} P_{L} \gamma^{\lambda} W_{2} P_{L} \gamma^{\nu}+W_{2} P_{L} \gamma^{\mu} W_{2} P_{L} \gamma^{\lambda} W_{2} \times \\
& P_{L} \gamma^{\nu}+W_{2} P_{L} \gamma^{\mu} W_{2} P_{L} \gamma^{\lambda} W_{1} P_{R} \gamma^{\nu}+W_{2} P_{L} \gamma^{\mu} W_{1} \times \\
& \left.P_{R} \gamma^{\lambda} W_{2} P_{L} \gamma^{\nu}+W_{2} P_{L} \gamma^{\mu} W_{1} P_{R} \gamma^{\lambda} W_{1} P_{R} \gamma^{\nu}\right] .
\end{aligned}
$$

Thus, taking into account the fact that $\left\{\gamma_{5}, \gamma^{\mu}\right\}=0$ and $\left(\gamma_{5}\right)^{2}=1$ and applying the following relation for trace $\operatorname{tr}\left(\gamma^{\lambda} \gamma^{\mu} \gamma^{\nu} \gamma^{\rho} \gamma_{5}\right)=4 i \epsilon^{\lambda \mu \nu \rho}$, we can write down the simple expression for self-energy tensor $\Pi^{\mu \nu \lambda}$ :

$$
\begin{aligned}
& \Pi^{\mu \nu \lambda}=i e^{2} \epsilon^{\lambda \mu \nu \rho} \int \frac{d^{4} p}{(2 \pi)^{4}} \frac{1}{\left(p^{2}-b^{2}\right)^{4}} \times \\
& b_{\rho}\left((p+b)^{4}+(p-b)^{4}\right)-p_{\rho}\left((p+b)^{4}-(p-b)^{4}\right) .
\end{aligned}
$$

Now, we can write our effective action in the form

$$
S_{e f f}^{(2)}[b, A]=\frac{1}{2} \int d^{4} x \epsilon^{\lambda \mu \nu \rho} k_{\rho} F_{\lambda \mu} A_{\nu},
$$

where the $k_{\rho}$-parameter can be expressed in the form

$$
\begin{aligned}
k_{\rho}= & 2 i e^{2} \int \frac{d^{4} p}{(2 \pi)^{4}}\left[\frac{\left(p^{2}-b^{2}\right) b_{\rho}-4(b \cdot p) p_{\rho}}{\left(p^{2}-b^{2}\right)^{3}}+\right. \\
& \left.\frac{4\left[\left(p^{2} b^{2}+(b \cdot p)^{2}\right) b_{\rho}-2 b^{2}(b \cdot p) p_{\rho}\right]}{\left(p^{2}-b^{2}\right)^{4}}\right] .
\end{aligned}
$$

Note that by power counting, the momentum integral in (27) involves finite terms and terms with logarithmic divergence.

\section{REGULARIZATION SCHEMES}

In the following we shall follow three different regularization schemes: $i$ ) the dimensional regularization, $i i$ ) the momentum cut-off regularization and a new one, $i i i$ ) the temperature regularization. As our results show, by using any of these schemes we got the same result for the coefficient of the induced Chern-Simons-like term. This implies that in an extended massless QED the induced Chern-Simons-like term is finite and determined under these regularization schemes.

\section{A. Dimensional regularization}

In this subsection, we shall first adopt a simpler regularization scheme such as dimensional regularization [34] in order to calculate the divergent integral. By assuming the usual statements for this case, we change the number of dimensions from 4 to $D$, and we also change $\int d^{4} p /(2 \pi)^{4}$ to $\left(\mu^{2}\right)^{\epsilon / 2}\left[\int d^{D} p /(2 \pi)^{D}\right]$, where $\mu$ is an arbitrary parameter that identifies the mass scale with $\epsilon=4-D$. The potential divergences in the momentum integration come from the first term of the expression (27). In order to carry out the calculations, we use the following relations

$$
\int \frac{d^{D} p}{(2 \pi)^{D}} \frac{1}{\left(p^{2}-m^{2}\right)^{2}}=\frac{i \Gamma(\epsilon / 2)}{(4 \pi)^{D / 2}\left(b^{2}\right)^{\epsilon / 2}},
$$

and

$$
\int \frac{d^{D} p}{(2 \pi)^{D}} \frac{p_{\mu} p_{\nu}}{\left(p^{2}-m^{2}\right)^{3}}=\frac{i \delta_{\mu \nu}}{4(4 \pi)^{D / 2}} \frac{\Gamma(\epsilon / 2)}{\left(b^{2}\right)^{\epsilon / 2}},
$$

in (27) so that the logarithmic divergence disappears. The resulting induced Chern-Simons-like coefficient is finite and exactly given by the value

$$
k_{\rho}=\frac{e^{2}}{8 \pi^{2}} b_{\rho} .
$$

\section{B. Momentum cut-off- $\Lambda$ regularization}

To develop calculations via momentum cut-off, we consider the expression (27) and change the $(3+1)$ dimensional Minkowski spacetime to a four-dimensional Euclidean space by performing the Wick rotation $x_{0} \rightarrow$ $-i x_{0}, p_{0} \rightarrow i p_{0}, b_{0} \rightarrow i b_{0}, d^{4} x \rightarrow-i d^{4} x$ and $d^{4} p \rightarrow i d^{4} p$. Thus, we have

$$
\begin{aligned}
& k_{\rho}=-\frac{2 i e^{2}}{(2 \pi)^{4}} \int_{-\infty}^{+\infty} \times \\
& d^{3} p \int_{-\infty}^{+\infty} d p_{0}\left[\frac{\left(p^{2}-b^{2}\right) b_{\rho}-4(b \cdot p) p_{\rho}}{\left(p^{2}-b^{2}\right)^{3}}+\right. \\
& \left.\frac{4\left[\left(p^{2} b^{2}+(b \cdot p)^{2}\right) b_{\rho}-2 b^{2}(b \cdot p) p_{\rho}\right]}{\left(p^{2}-b^{2}\right)^{4}}\right] .
\end{aligned}
$$

For the sake of simplicity, in (31) we shall choose only the time-like component of our Lorentz-symmetry violating parameter, such that $b_{\rho}=\left(b_{0}, 0\right)$, being $b_{0}$ a nonzero component. Such a simplification does not affect the four-vector coefficient of the induced Chern-Simons-like term. It simplifies our calculations without any loss of generality.

Taking into account the considerations above, we now write the expression (31) in spherical coordinates as

$$
\begin{aligned}
& k_{0}=-\frac{i e^{2} b_{0}}{2 \pi^{3}} \int_{0}^{\infty} d \vec{p} \vec{p}^{2} \int_{-\infty}^{+\infty} d p_{0} \times \\
& {\left[\frac{\vec{p}^{2}-3 p_{0}^{2}-b_{0}^{2}}{\left(\vec{p}^{2}+p_{0}^{2}-b_{0}^{2}\right)^{3}}+\frac{4 b_{0}^{2} \vec{p}^{2}}{\left(\vec{p}^{2}+p_{0}^{2}-b_{0}^{2}\right)^{4}}\right] .}
\end{aligned}
$$


The integrals over $p_{0}$ are finite and can be directly calculated. Hence, we have

$$
\begin{aligned}
k_{0}^{E} & =-\frac{5 i}{8} \frac{e^{2} b_{0}}{\pi^{2}} \int_{0}^{\infty} d \vec{p} \frac{b_{0}^{2} p^{4}}{\left(\vec{p}^{2}-b_{0}^{2}\right)^{7 / 2}}, \\
& =-\frac{5 i}{8} \frac{e^{2} b_{0}}{\pi^{2}} \int_{0}^{u=\Lambda / b_{0}} d u \frac{u^{4}}{\left(u^{2}-1\right)^{7 / 2}}, \\
& =\frac{i e^{2} b_{0}}{8 \pi^{2}} \frac{1}{\left(1-\frac{b_{0}^{2}}{\Lambda^{2}}\right)^{7 / 2}} .
\end{aligned}
$$

Therefore, taking the limit $\Lambda \rightarrow \infty$, we find (in Euclidean coordinates)

$$
k_{0}^{E} \rightarrow \frac{i e^{2}}{8 \pi^{2}} b_{0} .
$$

This result coincides with the coefficient (30), previously obtained via dimensional regularization.

\section{CHERN-SIMONS COEFFICIENT INDUCED UNDER FINITE TEMPERATURE EFFECTS}

The effect of finite temperature in the context of violating Lorentz symmetry has generated interesting studies in the literature [24, 33, 36, 37, 38]. In this case, let us assume that the system is in the state of the thermal equilibrium with a temperature $T=1 / \beta$. Therefore, we make the following substitutions: $p_{0} \equiv \omega_{0}=(n+1 / 2) \frac{2 \pi}{\beta}$ and $(1 / 2 \pi) \int d p_{0} \rightarrow \frac{1}{\beta} \sum_{n}$, where $\omega_{0}$ is the Matsubara frequency for fermions. Again, we consider the expression (27) and rewrite it in the framework of the imaginary time formalism as

$$
\begin{aligned}
k_{\rho}(\beta)= & \frac{-2 i e^{2}}{\beta} \sum_{n=-\infty}^{\infty} \int \frac{d^{3} \vec{p}}{(2 \pi)^{3}}\left[\frac{\left(p^{2}-b^{2}\right) b_{\rho}-4(b \cdot p) p_{\rho}}{\left(p^{2}-b^{2}\right)^{3}}+\right. \\
& \left.\frac{4\left[\left(p^{2} b^{2}+(b \cdot p)^{2}\right) b_{\rho}-2 b^{2}(b \cdot p) p_{\rho}\right]}{\left(p^{2}-b^{2}\right)^{4}}\right] .
\end{aligned}
$$

We can write the expression (35) for the time-like and space-like components of the Lorentz-symmetry violation parameter

$$
\begin{aligned}
k_{0}(\beta)= & \frac{2 i e^{2} b_{0}}{\beta} \int \frac{d^{3} \vec{p}}{(2 \pi)^{3}} \sum_{n=-\infty}^{\infty}\left[\frac{3}{\left(\omega_{0}^{2}+\vec{p}^{2}-b_{0}^{2}\right)^{2}}-\right. \\
& \left.\frac{4\left(\vec{p}^{2}-b_{0}^{2}\right)}{\left(\omega_{0}^{2}+\vec{p}^{2}-b_{0}^{2}\right)^{3}}-\frac{4 b_{0}^{2} \vec{p}^{2}}{\left(\omega_{0}^{2}+\vec{p}^{2}-b_{0}^{2}\right)^{4}}\right],
\end{aligned}
$$

and

$$
\begin{aligned}
k_{i}(\beta)= & \frac{-2 i e^{2}}{\beta} \int \frac{d^{3} \vec{p}}{(2 \pi)^{3}} \times \\
& \sum_{n=-\infty}^{\infty}\left[\frac{b_{i}}{\left(\omega_{0}^{2}+\vec{p}^{2}-b_{i}^{2}\right)^{2}}+\frac{4\left(b_{i}^{3}-(\vec{b} \cdot \vec{p})^{2} p_{i}\right)}{\left(\omega_{0}^{2}+\vec{p}^{2}-b_{i}^{2}\right)^{3}}+\right. \\
& \left.\frac{4\left[\left(b_{i}^{4}+(\vec{b} \cdot \vec{p})^{2}\right) b_{i}-2 b_{i}^{2}(\vec{b} \cdot \vec{p}) p_{i}\right]}{\left(\omega_{0}^{2}+\vec{p}^{2}-b_{i}^{2}\right)^{4}}\right]
\end{aligned}
$$

At this point, we can observe that both the 3 -momentum integrals and the sum over the Matsubara frequencies in (36) and (37) are convergent. This fact is interesting to our investigations from two points of view. The first, of course, concerns the study of high temperature effects on the parameter $k_{\mu}$. The second, and new one, is the possibility of understanding the temperature itself as a regulator of divergences. It means the inclusion of finite temperature in the theory can be also considered as a regularization scheme in the limit $\beta \rightarrow \infty(T \rightarrow 0)$. This is what we shall discuss just below.

\section{A. Temperature regularization}

- The time-like component

To calculate the time-like component, we consider the expression (36) and rewrite it in the following form

$$
\begin{aligned}
& k_{0}(\beta)=\frac{i e^{2} b_{0}}{\pi} \int \frac{d^{3} \vec{p}}{(2 \pi)^{3}} \sum_{n=-\infty}^{\infty}\left[\frac{3 a_{0}^{3}}{B_{0}^{3}\left(\left(n+\frac{1}{2}\right)^{2}+a_{0}^{2}\right)^{2}}-\right. \\
& \left.\frac{4 a_{0}^{5}}{B_{0}^{3}\left(\left(n+\frac{1}{2}\right)^{2}+a_{0}^{2}\right)^{3}}-\frac{4 a_{0}^{7} b_{0}^{2} \vec{p}^{2}}{B_{0}^{7}\left(\left(n+\frac{1}{2}\right)^{2}+a_{0}^{2}\right)^{4}}\right],
\end{aligned}
$$

where $a_{0}=B_{0} \beta / 2 \pi$ and $B_{0}=\left(\vec{p}^{2}-b_{0}^{2}\right)^{\frac{1}{2}}$. The series in the above expression can be easily summed over the Matsubara frequencies [35] to give

$$
\begin{aligned}
k_{0}(\beta)= & \frac{i e^{2} b_{0}}{\pi} \int \frac{d^{3} \vec{p}}{(2 \pi)^{3}} \times \\
& {\left[\frac{3 F_{1}\left(a_{0}\right)-4 F_{2}\left(a_{0}\right)}{\left(\vec{p}^{2}-b_{0}^{2}\right)^{\frac{3}{2}}}-\frac{4 b_{0}^{2} \vec{p}^{2} F_{3}\left(a_{0}\right)}{\left(\vec{p}^{2}-b_{0}^{2}\right)^{\frac{7}{2}}}\right], }
\end{aligned}
$$

where the functions $F_{1}\left(a_{0}\right), F_{2}\left(a_{0}\right)$ and $F_{3}\left(a_{0}\right)$ are given by

$$
\begin{aligned}
F_{1}\left(a_{0}\right)= & \frac{\pi}{2}\left[\tanh \left(a_{0} \pi\right)-a_{0} \pi \operatorname{sech}^{2}\left(a_{0} \pi\right)\right], \\
F_{2}\left(a_{0}\right)= & \frac{\pi}{8}\left[3 \tanh \left(a_{0} \pi\right)-a_{0} \pi \operatorname{sech}^{2}\left(a_{0} \pi\right) \times\right. \\
& \left.\left(3+2 a_{0} \pi \tanh \left(a_{0} \pi\right)\right)\right],
\end{aligned}
$$

and

$$
\begin{aligned}
F_{3}\left(a_{0}\right)= & \frac{\pi}{48}\left[15 \tanh \left(a_{0} \pi\right)+a_{0} \pi \operatorname{sech}^{2}\left(a_{0} \pi\right) \times\right. \\
& \left(6 a_{0}^{2} \pi^{2} \operatorname{sech}^{2}\left(a_{0} \pi\right)-12 a_{0} \pi \tanh \left(a_{0} \pi\right)-\right. \\
& \left.\left.4 a_{0}^{2} \pi^{2}-15\right)\right] .
\end{aligned}
$$

Let us now consider the limit $T \rightarrow 0$. In this limit $a_{0} \propto$ $\beta \rightarrow \infty$ and the functions above achieve $F_{1}\left(a_{0}\right)=\frac{\pi}{2}$, $F_{2}\left(a_{0}\right)=\frac{3 \pi}{8}$ and $F_{3}\left(a_{0}\right)=\frac{5 \pi}{16}$, as depicted in Fig. 2 . Now the expression (39) can be written as

$$
k_{0}=\frac{-5 i e^{2} b_{0}}{4} \int \frac{d^{3} \vec{p}}{(2 \pi)^{3}} \frac{b_{0}^{2} \vec{p}^{2}}{\left(\vec{p}^{2}-b_{0}^{2}\right)^{\frac{7}{2}}} .
$$


This integral is manifestly finite and does not require any further scheme of regularization. The exact value obtained from the expression (43) is precisely the same result as that found in (30) and (34) via dimensional and momentum cut-off regularization in the absence of temperature.

- The space-like component

To calculate the space-like component, we proceed just as in the earlier case. We consider the expression (37) and rewrite it in the following form

$$
\begin{aligned}
k_{i}(\beta)= & \frac{-i e^{2}}{\pi} \int \frac{d^{3} \vec{p}}{(2 \pi)^{3}} \sum_{n=-\infty}^{\infty} \times \\
& {\left[\frac{b_{i} a_{i}^{3}}{B_{i}^{3}\left(\left(n+\frac{1}{2}\right)^{2}+a_{i}^{2}\right)^{2}}+\frac{\left[b_{i}^{3}-(\vec{b} \cdot \vec{p}) p_{i}\right] a_{i}^{5}}{B_{i}^{5}\left(\left(n+\frac{1}{2}\right)^{2}+a_{i}^{2}\right)^{3}}+\right.} \\
& \left.\frac{4\left[\left(b_{i}^{4}+(\vec{b} \cdot \vec{p})^{2}\right) b_{i}-2 b_{i}^{2}(\vec{b} \cdot \vec{p}) p_{i}\right] a_{i}^{7}}{B_{i}^{7}\left(\left(n+\frac{1}{2}\right)^{2}+a_{i}^{2}\right)^{4}}\right]
\end{aligned}
$$

where $a_{i}=B_{i} \beta / 2 \pi$ and $B_{i}=\left(\vec{p}^{2}-b_{i}^{2}\right)^{\frac{1}{2}}$. Here, just as in the time-like case, the sum over the Matsubara frequencies can be directly calculated to give

$$
\begin{aligned}
k_{i}(\beta)= & \frac{-i e^{2}}{\pi} \int \frac{d^{3} \vec{p}}{(2 \pi)^{3}} \times \\
& {\left[\frac{b_{i} F_{1}\left(a_{i}\right)}{\left(\vec{p}^{2}-b_{i}^{2}\right)^{\frac{3}{2}}}+\frac{4\left[b_{i}^{3}-(\vec{b} \cdot \vec{p}) p_{i}\right] F_{2}\left(a_{i}\right)}{\left(\vec{p}^{2}-b_{i}^{2}\right)^{\frac{5}{2}}}+\right.} \\
& \left.\frac{4\left[\left(b_{i}^{4}+(\vec{b} \cdot \vec{p})^{2}\right) b_{i}-2 b_{i}^{2}(\vec{b} \cdot \vec{p}) p_{i}\right] F_{3}\left(a_{i}\right)}{\left(\vec{p}^{2}-b_{i}^{2}\right)^{\frac{7}{2}}}\right]
\end{aligned}
$$

The functions $F_{1}\left(a_{i}\right), F_{2}\left(a_{i}\right)$ and $F_{3}\left(a_{i}\right)$ have the same functional form given by the expressions (40), (41) and (42). Now we can use the identity $p_{i} p_{j}=\frac{\vec{p}^{2}}{3} \delta_{i j}$ in (45), to obtain the simpler equation

$$
\begin{aligned}
k_{i}(\beta)= & \frac{-i e^{2} b_{i}}{2} \int \frac{d^{3} \vec{p}}{(2 \pi)^{3}}\left[\frac{F_{1}\left(a_{i}\right)-\frac{4}{3} F_{2}\left(a_{i}\right)}{\left(\vec{p}^{2}-b_{i}^{2}\right)^{\frac{3}{2}}}+\right. \\
& \left.\frac{8}{3}\left[\frac{b_{i}^{2} F_{2}\left(a_{i}\right)}{\left(\vec{p}^{2}-b_{i}^{2}\right)^{\frac{5}{2}}}+\frac{b_{i}^{4} F_{3}\left(a_{i}\right)}{\left(\vec{p}^{2}-b_{i}^{2}\right)^{\frac{7}{2}}}\right]\right] .
\end{aligned}
$$

By using again the limit $T \rightarrow 0$, we have

$$
k_{i}=-i e^{2} b_{i} \int \frac{d^{3} \vec{p}}{(2 \pi)^{3}}\left[\frac{b_{i}^{2}}{\left(\vec{p}^{2}-b_{i}^{2}\right)^{\frac{5}{2}}}+\frac{5}{6} \frac{b_{i}^{4}}{\left(\vec{p}^{2}-b_{i}^{2}\right)^{\frac{7}{2}}}\right] .
$$

In this case we also obtain only finite integrals that does not require any further scheme of regularization. Thus, similarly to what happen with the time-like component, the exact value obtained from the expression (47) is the same as the result found in (30) and (34) in the absence of temperature.

In summary, we find that the induced Chern-Simonslike coefficient that is temperature regularized has the following time-like and space-like components

$$
k_{0}=\frac{i e^{2}}{8 \pi^{2}} b_{0}, \quad k_{i}=\frac{i e^{2}}{8 \pi^{2}} b_{i},\left(a_{0} \rightarrow \infty \text { or } T \rightarrow 0\right) .
$$

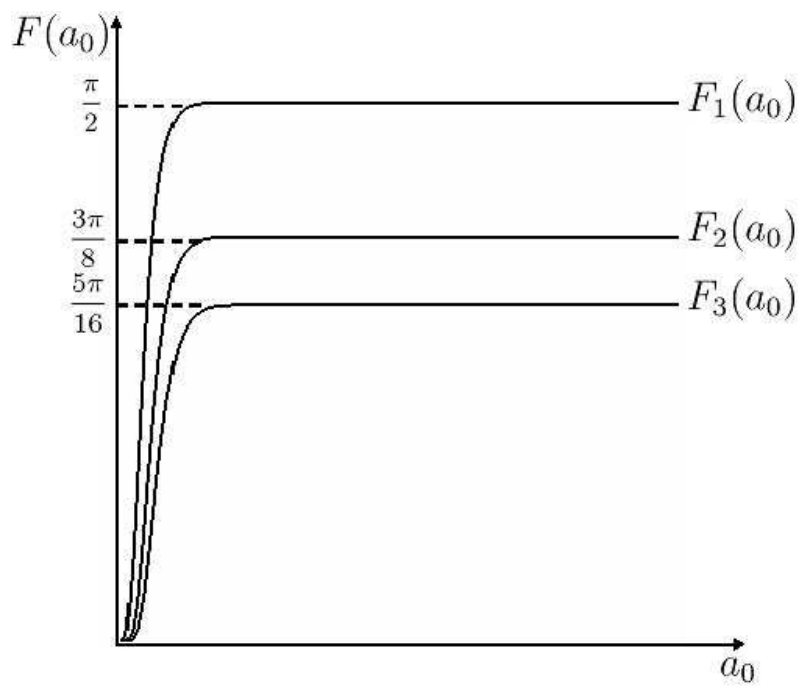

FIG. 2: The functions $F_{1}\left(a_{0}\right), F_{2}\left(a_{0}\right)$, and $F_{3}\left(a_{0}\right)$. At zero temperature $\left(\beta \rightarrow \infty, a_{0} \rightarrow \infty\right)$, they tend to distinct values, $\frac{\pi}{2}, \frac{3 \pi}{8}$, and $\frac{5 \pi}{16}$, respectively.

\section{CONCLUSIONS}

In this paper we have shown that the induced ChernSimons-like term in an extended massless QED is finite and determined at least in the framework of the three regularization schemes we have used: $i$ ) the dimensional regularization, $i i$ ) the momentum cut-off regularization and a new one, iii) the temperature regularization. A similar observation concerning determined Chern-Simonslike term in extended massless QED has also been done in the Ref. [10]. We state here that this has to do with the fact that one can relate an extended masless QED in $3+1$ dimensions with a massive QED in $2+1$ dimensions where the induced Chern-Simons term is finite and determined since the theory is finite. By dimensional reduction from $3+1$ down to $2+1$ dimensions, one component of the $3+1$ Chern-Simons-like term gives the coefficient of the $2+1$ Chern-Simons term. Since this term comes from a finite theory, it should be a finite and determined term. This automatically implies that at least one component of the four-vector coefficient $k_{\mu}$ of the $3+1$ Chern-Simons-like term, i.e. the $2+1$ Chern-Simons counterpart, is also finite and determined. Although this fact may guarantee that only one component is well determined, in our explicit calculations, in order to induce the Chern-Simonslike term, we have found that all components are finite and well determined.

\section{Acknowledgments}

We would like to thank CNPq, CAPES, PNPD/PROCAD-CAPES, and FAPERJ for partial financial support. 
[1] D. Colladay, V.A. Kostelecký, Phys. Rev. D 55 (1997) 6760; D 58 (1998) 116002.

[2] V. A. Kostelecky and S. Samuel, Phys. Rev. D39, 683 (1989).

[3] S. Carroll, G.Field, and R. Jackiw, Phys. Rev. D41, 1231 (1990).

[4] M. Goldhaber and V. Trimble, J. Astrophys. Astr. 17, 17 (1996); S. Carrol and G. Field, Phys. Rev. Lett, 79, 2394 (1997).

[5] S. Coleman and S. L. Glashow, Phys. Lett. 405B, 249, (1997); Phys. Rev. D59, 116008 (1998).

[6] D. Colladay and V. A. Kostelecky, Phys. Rev. D58, 116002 (1998).

[7] J. M. Chung and P. Oh, Phys. Rev. D 60, 067702 (1999).

[8] R. Jackiw and V. A. Kostelecky, Phys. Rev. Lett. 82, 3572 (1999).

[9] R. Jackiw, Nucl. Phys. Proc. Suppl. 108, 30 (2002).

[10] M. Perez-Victoria, Phys. Rev. Lett. 83, 2518 (1999).

[11] J. M. Chung, Phys. Rev. D60, 12790 (1999); J. M. Chung, Phys. Lett. B461, 138 (1999).

[12] A. A. Andrianov, P. Giacconi and R. Soldati, JHEP 0202, 030 (2002).

[13] O. Bertolami and C.S. Carvalho, Phys. Rev. D61, 103002 (2000).

[14] M. Chaichian, W.F. Chen and R. Gonzalez Felipe, Phys. Lett. B503, 215 (2001).

[15] B. Altschul, Phys. Rev. D69, 125009 (2004).

[16] B. Altschul, Phys. Rev. D70, 101701 (2004).

[17] O. Bertolami and J.G. Rosa, Phys. Rev. D71, 097901 (2005).

[18] R. Lehnert and R. Potting, Phys. Rev. Lett. 93, 110402 (2004).

[19] E. Kant and F.R. Klinkhamer, Nucl. Phys. B731, 125 (2005).

[20] B. Feng, M. Li, J.-Q. Xia, X. Chen and X. Zhang, Phys. Rev. Lett. 96, 221302 (2006).

[21] H. Belich, J. L. Boldo, L. P. Colatto, J.A. Helayel-Neto and A. L. M. A. Nogueira, Phys. Rev. D68, 065030 (2003).

[22] A.J. Hariton and R. Lehnert, Phys. Lett. A367, 11 (2007).
[23] N.M. Barraz Jr., J.M. Fonseca, W.A. Moura-Melo, J.A. Helayel-Neto, Phys. Rev. D76, 027701 (2007).

[24] F. A. Brito, J. R. Nascimento, E. Passos, A. Yu. Petrov, JHEP 06, 016 (2007).

[25] R. Jackiw, Int. J. Mod. Phys. B 14, 2011 (2000); J. M. , Phys. Rev. D60, 127901 (1999).

[26] G.Bonneau, Nucl. Phys. B593, 398 (2001); G. Bonneau, Lorentz and CPT violations in QED: a short comment on recent controversies hep-th/0109105.

[27] W.F. Chen, Issues on Radiatively Induced Lorentz and CPT Violation in Quantum Electrodynamics hep-th/0106035.

[28] M. Perez-Victoria, JHEP 04, 032 (2001).

[29] G. Bonneau, Nucl.Phys. B764, 83 (2007).

[30] H. Belich, M.M. Ferreira Jr, J.A. Helayel-Neto, M.T.D. Orlando, Phys. Rev. D67, 125011 (2003); Erratum-ibid. D69 109903 (2004).

[31] A. P. B. Scarpelli, M. Sampaio, M. C. Nemes and B. Hiller, Eur. Phys. J. C 56, 571 (2008).

[32] I. J. R. Aitchison and C. M. Fraser, Phys. Lett. B 146, 63 (1984); Phys. Rev. D 31, 2605 (1985); C. M. Fraser, Z. Phys. C 28, 101 (1985); A. I. Vainshtein, V. I. Zakharov, V. A. Novikov, and M. A. Shifman, Yad. Fiz. (Sov. J. Nucl. Phys.) 39, 77 (1984); J. A. Zuk, Phys. Rev. D 32, 2653 (1985); M. K. Gaillard, Nucl. Phys. B 268, 669 (1986); A. Das and A. Karev, Phys. Rev. D 36, 623 (1987); K. S. Babu, A. Das, and P. Panigrahi, Phys. Rev. D 36, 3725 (1987).

[33] F. A. Brito, T. Mariz, J. R. Nascimento, E. Passos and R. F. Ribeiro, JHEP 10, 019 (2005).

[34] G. 't Hooft and M.J.G. Veltman, Nucl.Phys. 44 (1972) 189.

[35] A. R. Prudnikov, Yu. A. Brychkov and O. I. Marichev, Integrals and Series (in Russian), Moscow, 1981.

[36] L. Cervi, L. Griguolo and D. Seminara, Phys. Rev. D 64, 105003 (2001).

[37] D. Ebert, V. C. Zhukovsky and A. S. Razumovsky, Phys. Rev. D 70, 025003 (2004).

[38] M. Gomes, J. R. Nascimento, E. Passos, A. Y. Petrov and A. J. da Silva, Phys. Rev. D 76, 047701 (2007). 